\title{
Ambientes de aprendizaje basados en herramientas web para el desarrollo de competencias TIC en la docencia
}

\author{
María Mercedes- Martínª, César Augusto Hernández-Suarez ${ }^{\text {b*, }}$ \\ Sonia Maritza Mendoza-Lizcano ${ }^{c}$.

\begin{abstract}
${ }^{a}$ Magíster en Procesos Educativos Mediados por Tecnologías. Profesora Adjunta de la Carrera de Ciencias de la Educación-Universidad Nacional de La Plata (Argentina) (1D. bagister en Enseñanza de las Ciencias Básicas. Docente de la Facultad de Educación, Artes y Humanidades - Universidad Francisco de Paula Santander. (D)
\end{abstract} \\ 'Docente de la Facultad de Ciencias Básicas - Universidad Francisco de Paula Santander (ID)
}

Forma de citar: Martín, M., Hernández, C. \& Mendoza, S. (2017). Ambientes de aprendizaje basados en herramientas web para el desarrollo de competencias TIC en la docencia. Perspectivas, 2(1). 97-104

Recibido: julio 23 de 2016

Aceptado: octubre 15 de 2016

\section{Palabras clave}

Ambiente de aprendizaje, Medios

y Tecnologías de

Información y

Comunicación (MTIC),

Web 2.0, competencias

TIC

\begin{abstract}
Resumen: El presente artículo realiza una reflexión sobre un nuevo entorno educativo donde docentes adopten estrategias pedagógicas incluyan el uso de Medios y Tecnologías de Información y Comunicación (MTIC) como las herramientas Web 2.0 para diseñar ambientes de aprendizajes que permitan construir y compartir conocimientos. El escrito hace un análisis sobre la formación del docente orientado a innovar y experimentar con las TIC y reflexionar sobre su uso, favoreciendo el desarrollo y la adquisición de diferentes competencias TIC que le permitan utilizar las herramientas y servicios procedentes de la Web 2.0, convirtiéndose en un medio más dentro del aula. Se concluye con una visión general de la necesidad y conveniencia de implementar y usar estas herramientas y servicios de comunicación e información para una real inclusión en la Sociedad del Conocimiento.
\end{abstract}

\footnotetext{
*Autor para correspondencia cesaraugusto@ufps.edu.co

Universidad Francisco de Paula Santander, Cúcuta.
} 


\section{Keywords}

Learning Environment, Mediaand Information and Communications Technologies (MICT), Web 2.0, ICT competences

\section{Palavras chave \\ Ambiente de aprendizagem, Mídia e Tecnologia da Informação e Comunicação (MICT), Web 2.0, competências em TIC}

\section{Learning environments based on web tools: concerning the development of ICT skills in teaching}

\begin{abstract}
This article considers the possibility of a new educational environment in which teachers can embrace the teaching strategies that incorporate the use of Media, Information and Communications Technologies (MICT), such as Web 2.0 tools, in order to design learning environments satisfactory for the generation and sharing of knowledge. This paper analyzes the requirement of teacher training focused on the innovation and experimentation with ICTs and reflects on the use of these technologies, favoring the development and acquisition of different ICT skills which will allow teachers to use the tools and services stemming from Web 2.0, which in turn will transform into another teaching mechanism. The paper concludes with a general overview of the convenience and necessity of implementing these communication and information technologies for effective inclusion into the knowledge society.
\end{abstract}

\section{Aprender ambientes ferramentas baseadas na Web para o desenvolvimento de competências em TIC no ensino}

Resumo: Este artigo faz uma reflexão sobre um novo ambiente educacional onde os professores adotam estratégias pedagógicas incluem o uso de mídia e Informação e Comunicação Tecnologia (MICT) e ferramentas da Web 2.0 para projetar ambientes que construir e compartilhar conhecimentos aprendendo. Escrito uma análise da formação de professores orientada para inovar e experimentar com as TIC e refletir sobre a sua utilização, promovendo o desenvolvimento e aquisição de várias competências em TIC que permitem ferramentas e serviços de uso da Web 2.0, tornando-se outro meio na sala de aula. Ele conclui com uma visão geral sobre a necessidade e conveniência de implementar e usar essas ferramentas e serviços de comunicação e informação para a inclusão real na Sociedade do Conhecimento.

\section{Introducción}

En el siglo XXI, se espera que cada vez más las personas hagan utilización de las TIC para acceder y manipular la información. Para estar plenamente preparado $\mathrm{y}$ funcionar productivamente en una sociedad orientada a las TIC, las personas deben desarrollar no sólo conocimientos de informática básica, sino también la competencia en el uso de una variedad de herramientas TIC para resolver problemas, tomar decisiones, y generar nuevos conocimientos. El desarrollo de estas competencias es responsabilidad de los docentes. Sin embargo, muchos de los educadores carecen de las competencias necesarias para asumir la integración de las TIC en la práctica pedagógica. Es partir de las reflexiones anteriores que surgen preguntas como: ¿Qué competencias son necesarias para utilizar las 
TIC?, ¿Cómo las adquirirán los docentes?, ¿Cómo se utilizan actualmente las TIC y para qué fines? ¿Cómo actualizar la formación del docente? ¿Cómo se está realizando su formación en TIC? ¿Y en web 2?0? entre otras.

\subsection{La Web 2.0.}

El término fue utilizado por primera vez por Darcy DiNucci en 1999, en el artículo "Fragmented future", fue acuñado por Dale Dougherty de la editorial Tim O’Reilly Media en el año 2004. El término surgió para referirse a nuevos sitios web que se diferenciaban de los sitios web más tradicionales englobados bajo la denominación Web 1.0; es una denominación que se refiere a una segunda generación en la historia de los sitios web. Por tanto, Web 2.0 refiere a la transición del html estático de páginas web, a webs más dinámicas que están más organizadas y basadas en aplicaciones de servicio a los usuarios incluyendo una importante función comunicativa abierta, con énfasis en las webs sustentadas en comunidades de usuarios.

La Web 2.0 o también llamada Web social engloba un gran número de espacios web basados en el principio de una comunidad de usuarios. Además del avance tecnológico experimentado, Web 2.0 no es solo eso, tecnología, sino que está ligada a una actitud de los usuarios de estas herramientas, ya que la mayoría tiene un componente de red social, es decir, que se agrupan usuarios con intereses y finalidades comunes.

Abarca una amplia variedad de redes sociales, blogs, wikis y servicios multimedia interconectados cuyo propósito es el intercambio ágil de información entre los usuarios y la colaboración en la producción de contenidos. Así se pone al alcance de cualquier usuario de Internet nuevas herramientas, de texto, imagen, vídeo, sonido, entre otras. Estas aplicaciones, muy fáciles de utilizar y gratuitas, solo requieren registrarse como usuario disponiendo de un e-mail, permiten que todos puedan publicar y compartir contenidos en varios formatos en la web con sólo disponer de acceso a Internet. Según de la Torre (2006, p.2),'Internet ha pasado de ser un espacio de lectura a ser de lectura-escritura".

Por lo tanto, no hay definición consensuada, ya que se trata de una etiqueta para nombrar una tendencia. Según Ortiz de Zárate (2008, p.18)_es "un fenómeno social en relación con la creación y distribución de contenidos en Internet, caracterizado por la comunicación abierta, la descentralización de autoridad, la libertad de compartir y usar, dentro de un enfoque que trata a las relaciones humanas como conversaciones". También se puede entender como 2.0 "todas aquellas utilidades y servicios de Internet que se sustentan en una base de datos, la cual puede ser modificada por los usuarios del servicio, ya sea en su contenido (añadiendo, cambiando o borrando información o asociando datos a la información existente), pues bien, en la forma de presentarlos, o en contenido y forma simultáneamente" Ribes (2007, p.2).

La Web 2.0 se caracteriza principalmente por la participación del usuario como contribuidor activo y no solo como espectador de los contenidos de la Web (usuario pasivo). Esto queda reflejado en aspectos como el auge de los blogs y las redes sociales, la creación de webs por los usuarios, usando plataformas de auto-edición, el etiquetado colectivo (folcsonomía, marcadores sociales...), la importancia del long tail, el beta perpetuo (se mejora continuamente): la Web 2.0 se inventa permanentemente y las aplicaciones web dinámicas.

La Web 2.0 se utiliza una serie de herramientas y servicios, algunas aplicaciones útiles en docencia de estas herramientas se muestran a continuación:

1. Blogs: Un blog es un espacio web personal en el que su autor (puede haber varios autores autorizados) puede escribir cronológicamente artículos, noticias... (Con imágenes videos y enlaces), pero además es un espacio colaborativo donde los lectores también pueden escribir sus comentarios a cada uno de los artículos (entradas/ post) que ha realizado el autor. La información se organiza en artículos ordenados cronológicamente, páginas, enlaces y comentarios. La blogosfera es el conjunto de blogs que hay en internet. Es el nodo 
más abundante en la Web 2.0. Como servicio para la creación de blogs destacan Wordpress.com y Blogger.com

2. Wikis: En hawaiano "wiki” significa: rápido, informal. Una wiki es un espacio web corporativo, organizado mediante una estructura hipertextual de páginas (referenciadas en un menú lateral), donde varias personas elaboran contenidos de manera asíncrona. No están tan extendidos como los blogs porque demandan mayor nivel de compromiso en la construcción de contenidos. Se puede utilizar para crear la documentación de un proyecto de forma colaborativa: glosarios, enciclopedias, manuales, enlaces, etc. El espacio wiki por excelencia es la enciclopedia Wikipedia (http://es.wikipedia.org/). Hay diversos servidores de wikis gratuitos.

3. Redes sociales: Sitios web donde cada usuario tiene una página donde publica contenidos y se comunica con otros usuarios. Ejemplos: Facebook, Twitter, Tuenti, Hi5, MySpace, Instagram, entre otras. También existen redes sociales profesionales, dirigidas a establecer contactos dentro del mundo empresarial (LinkedIn, Xing, e Conozco, Neurona...).

\section{Entornos para compartir recursos:} Entornos que nos permiten almacenar recursos o contenidos en Internet, compartirlos y visualizarlos cuando nos convenga. Constituyen una inmensa fuente de recursos y lugares donde publicar materiales para su difusión mundial. Existen de diversos tipos, según el contenido que albergan o el uso que se les da:

- Documentos: Google Drive y Office Web Apps (OneDrive), en los cuales podemos subir nuestros documentos, compartirlos y modificarlos.

- Videos: Youtube, Vimeo, Dailymotion, Dalealplay... Contienen miles de vídeos subidos y compartidos por los usuarios.

- Fotos e imagenes: Picasa, Flickr, Instagram... Permiten disfrutar y compartir las fotos también tenemos la oportunidad de organizar las fotos con etiquetas, separándolas por grupos como si fueran álbumes, podemos seleccionar y guardar aparte las fotos que no queremos publicar.
- Agregadores de noticias: Digg, Reddit, Menéame, Divoblogger... Noticias de cualquier medio son agregadas y votadas por los usuarios.

- Almacenamiento online: Dropbox, Google Drive, SkyDrive

- Presentaciones: Prezi, Slideshare.

5. Gestores CMS (Content Management System o Sistema de Gestión de Contenidos): Se suelen utilizar para crear sitios web institucionales o corporativos. Permiten la publicación colaborativa (roles) en línea de las noticias que afectan a una comunidad. Basan la organización de los artículos en secciones (cajones) y categorías (carpetas dentro de esos cajones). El uso de CMS deja atrás los sitios web estáticos HTML diseñados con FrontPage, Dreamweaver, etc y que se actualizaban subiendo sus páginas mediante un cliente FTP. Algunos ejemplos de CMS gratuitos son Joomla (http://www. joomlaspanish.org/) o Drupal (http://drupal.org.es/).

6. Plataforma e-learning: Se denominan sistemas LMS (Learning Management System o Sistema de Gestión del Aprendizaje) y se utilizan para crear entornos de educación virtual. El LMS más utilizado actualmente es Moodle (http://moodle. org/). El profesor/a crea un curso que consiste en una agenda de contenidos y actividades que el alumno realiza siguiendo la progresión establecida. El sistema se complementa con unas herramientas de comunicación (mensajería interna y foros) y con un subsistema de seguimiento de la actividad del estudiante. Muchas universidades e instituciones públicas educativas han abandonado el desarrollo de sus propias plataformas de e-learning en favor del uso de Moodle. Tanto los CMS como LMS se han clasificado como sitios "Web 1.5" porque admiten un uso 1.0 ó 2.0 en función de cómo sus administradores organicen la participación de los usuarios.

\subsection{La escuela 2.0.}

Las dotaciones en infraestructura tecnológica que se están haciendo a nivel educativo pretenden que las instituciones educativas incorporen los avances de la sociedad del conocimiento. Las nuevas 
herramientas, tales como los tablets PC, mejoran las oportunidades para la enseñanza y favorecen la realización de actividades cooperativas a partir del uso de instrumentos como las wikis, los blogs, entre otros junto a la implementación de estrategias colaborativas como las webquests permitiendo el desarrollo de numerosas habilidades cognitivas, así como la adquisición de las competencias TIC.

En el ámbito educativo, la utilización de las herramientas de la Web 2.0 han facilitado a docentes y estudiantes el acceso a contenidos educativos de calidad, así como la selección, clasificación, integración e interrelación de los más adecuados para favorecer la construcción del conocimiento de forma colaborativa.

Todo ello está potenciando que se generen nuevas y enriquecedoras experiencias, proyectos e iniciativas educativas apoyadas en las TIC que están suponiendo cambios cualitativos en la forma de presentar y acceder a la información, y abriendo nuevos cauces de participación e interacción permitiendo el planteamiento de actividades colaborativas que favorezcan la adquisición del conocimiento como resultado tanto del esfuerzo personal y del grupo, al propiciar la participación comprometida y el diálogo activo entre todos, y al compartir información, ideas y/o experiencias (Del Moral y Villalustre, 2009).

Todos estos cambios que se han ido generando en la sociedad y los avances producidos con la Web 2.0 han provocado cambios sustanciales en las funciones y los roles asumidos por el nuevo docente, adquiriendo un papel más dinámico, facilitador de los aprendizajes, y no un mero transmisor de contenidos.

Por su parte, el perfil del nuevo estudiante procedente de un mundo digital que demanda, tal y como manifiestan Gisbert y Cabero (2007), nuevas y diferentes acciones formativas apoyadas en el uso de las tecnologías Web 2.0, y consecuentemente, ello exige cambios en el perfil del docente y en su acción formativa, dando lugar a la figura que se ha venido a denominar profesor 2.0.
En este sentido, y con objeto de delinear esa nueva figura, "el docente 2.0", y la "docencia 2.0" que designa a un conjunto de técnicas apoyadas por servicios Web 2.0 utilizados en ámbitos docentes, queentra a formar parte del escenario educativo, se precisa favorecer la adquisición y el desarrollo de determinadas competencias, fundamentalmente de orden tecnológico, para que sea capaz de hacer frente a las nuevas situaciones de aprendizaje que se generan con la utilización de las TIC y de las ya mencionadas herramientas de la Web 2.0.

\subsection{La Web 3.0.}

La Web 2.0 significó la aparición de redes sociales la Web 3.0, que centra su objetivo en la inteligencia artificial y a la innovación tecnológica.

El termino Web 3.0,también conocido como Web Semántica, fue acuñado por Berners-Lee, Hendler, y Lassila (2001), es una extensión de la web actual en la que se da a la información un significado bien definido, lo que permite que las computadoras y la gente trabajen en cooperación. Busca introducir una serie de lenguajes y procedimientos que puedan interpretar ciertas características del usuario con el objetivo de ofrecer una interfaz más personalizada. Si bien no existe un consenso sobre la definición de este nuevo término y sus implicancias en el uso de la red, sí existen ciertas características que nos ayudan a darle forma a este concepto, tales como las de inteligencia (búsquedas inteligentes), sociabilidad (la evolución de las redes sociales), ubicuidad (conectividad a través de más dispositivos), tridimensionalidad (espacios tridimensionales, web geoespacial) entre otras (Santos, Nava y Godoy, 2009).

La Web Semántica tiene aplicaciones en entornos de educación y entrenamiento como el E-learning, simulaciones educativas ("aprender haciendo"); entornos de colaboración semántica; servicios de bibliotecas digitales; adaptación rápida de contenido; automatización de encuestas, entre otras. (Davis, 2008, p. 23). 
De este modo, las tecnologías inmersas en la Web 3.0, potenciarán el desarrollo de sistemas colaborativos, permitiendo crear sistemas tutoriales, realizar búsquedas y manejar contenido a partir del ensamblaje autónomo de piezas informacionales con lógica acoplable (Ramírez, Peña, 2011).

\subsection{Competencias TIC de los docentes.}

Las competencias se definen como "la capacidad de responder a demandas complejas y llevar a cabo tareas diversas de forma adecuada, suponiendo una combinación de habilidades prácticas, conocimientos, motivación, valores éticos, actitudes, emociones y otros componentes sociales y de comportamiento que se movilizan conjuntamente para lograr una acción eficaz" (Salganik L.H., Rychen D.S., Moser U. \& Konstant J.W., 1999).

El Ministerio de Educación Nacional de Colombia (MEN) en el año 2013, enuncia las competencias TIC en las que se debería potenciarla formación docente; las competencias consisten en habilidades relacionadas con las TIC, agrupados en cinco dimensiones generales: (1) Tecnológicas, (2) Pedagógicas, (3) Comunicativas, (4) Investigativas, y (5) de Gestión

Un amplio análisis de las competencias TIC para docentes del MEN (2013) lo hacen Hernández, Arévalo y Gamboa (2016) y Hernández, Ayala y Gamboa (2016) donde se pueden definir como un conjunto de estándares y descriptores de competencias que definen el dominio en el uso de las TIC en el aula.

Las competencias en TIC se basan en el uso de herramientas y servicios técnicos para favorecer la inserción de las personas en la sociedad del conocimiento. Incluyen cualquier tecnología que ayuda a producir, manipular, almacenar, comunicar, y/o difundir información. Esto significa que la competencia de las TIC es muy importante para mejorar la comunicación en el proceso de aprendizaje y enseñanza.
Importancia de las competencias TIC para los docentes. La práctica pedagógica es una actividad compleja; los profesores competentes aplican conjuntos amplios, profundos, e integrados de conocimientos y habilidades en la medida que planifican, implementan y revisan su práctica pedagógica. La adquisición de competencias TIC debe ser conectada al desarrollo de una gama más amplia de competencias docentes.

Algunos organismos multilaterales y países se han ocupado activamente en el problema de la integración de las TIC a la educación y a la docencia y han publicado recientemente un conjunto de estándares TIC. La UNESCO, ISTE (International Society Technology Education), QTS (Standards for the award of Qualified Teacher Status - Reino Unido), European pedagogical ICT (Comunidad Europea), ICEC (Instituto Canario de Evaluación y Calidad Educativa de Canarias) y la Universidad de Murcia $(2010,2011)$ de España y la Red Enlaces (Chile) entre otros.

Estrategias de formación en TIC para docentes. Una breve descripción de las posibles estrategias de formación es la siguiente:

- Motivar a los docentes a adquirir las habilidades necesarias: Para muchos docentes, el tener acceso a las TIC inicialmente no es visto como un beneficio. Según ellos, las TIC demandan mucho tiempo, son herramientas que no pidieron y que no saben cómo utilizar. Otros creen que sin las TIC están haciendo un buen trabajo en el aula y se preguntan cómo contribuirán a mejorar su práctica de aula. Sin embargo, otros docentes, le dan la bienvenida a las tecnologías y están motivados por aprender cómo usarlas.

- Proporcionar un apoyo sostenido a los docentes en el uso de las TIC: Es muy importante que los docentes adquieran conocimientos y habilidades en el uso de las tecnologías. Una vez que los docentes adquieran dichos conocimientos y utilicen las TIC, es necesario proporcionar los medios y apoyo para que los docentes continúen usándolas. Es decir, la sola formación inicial de los docentes no será 
adecuada para garantizar que seguirá utilizando la infraestructura tecnológica con la que disponen.

\section{Conclusiones}

Ante todos estos cambios, avances y grandes posibilidades que se presentan, en el ámbito educativo los docentes no pueden "hacer oídos sordos", sino que deben por su propio bien y sobre por el de los estudiantes, que en definitiva está siendo y va a ser el protagonista de todos estos acontecimientos, estar al día, estar informados, formados para educarlos y guiarlos en todo su proceso educativo y formativo, en la construcción de su aprendizaje, en el que las TIC, Internet y su evolución (Web 2.0, 3.0), son cada vez más protagonista de sus vidas.

Es incuestionable que la sociedad actual exige a los docentes una actualización constante y permanente para enseñar y formar a los estudiantes, no sólo apoyándose en el uso de todas las herramientas y recursos a su alcance, especialmente en las TIC, sino que debe fomentar entre sus estudiantes el uso de las mismas ofreciéndoles experiencias didácticas enriquecedoras.

Por tanto, la formación del docente debe ir encaminada a favorecer la adquisición $y$ consolidación de las competencias TIC necesarias para lograr una idónea integración curricular de las TIC, con fin de optimizar los aprendizajes de los estudiantes y de alcanzar una mejora cualitativa en las estrategias de enseñanza.

\section{Referencias bibliográficas}

Berners-Lee, T., Hendler, J. y Lassila, O. (2001). The Semantic Web. A new form of Web content that is meaningful to computers will unleash a revolution of new possibilities. Scientific American.

Davis, M. (2008). Project 10X's. Semantic Wave 2008 Report: Industry Roadmap to Web 3.0 \& Multibillion Dollar Market Opportunities. Executive summary. Recuperado de http://www.eurolibnet.eu/
files/REPOSITORY/20090507165103 SemanticWaveReport2008.pdf

De la Torre, A. (2006).Web Educativa 2.0. Edutec. Revista Electrónica de Tecnología Educativa, 20.

Del Moral, M. E. Y villalustre, L. (2009): Evaluación de prácticas docentes universitarias desarrolladas en entornos virtuales. Pixel-Bit: Revista de Medios y Educación, 34, 151-163.

DiNucci, D. (1999). Fragmented future. Print Magazine, 4, 32. Recuperado de http://darcyd. com/fragmented_future.pdf

Gisbert, M. Y Cabero, J. (2007): El papel del profesor $\mathrm{y}$ el estudiante en los entornos tecnológicos de formación. Tecnología Educativa. Madrid: McGrawHill.

Hernández Suárez, C. A, Ayala García, E. T, y Gamboa Suárez, A. A. (2016). Modelo de competencias TIC para docentes: Una propuesta para la construcción de contextos educativos innovadores y la consolidación de aprendizajes en educación superior. Katharsis, 22, 221-265.

Hernández Suárez, C. A.; Arévalo Duarte, M. A.; Gamboa Suárez, A. A. (2016). Competencias TIC para el desarrollo profesional docente en educación básica. Praxis \& Saber, 7(14), 41 - 69. doi: http://dx.doi.org/10.19053/22160159.5217.

Instituto Canario de Evaluación y Calidad Educativa. (2004). Competencias básicas en las tecnologías de la información y la comunicación (TIC). ICEC, Gobierno de Canarias. Consejería de Educación, Cultura y Deportes.

Internacional Society for Technology in Education. (2008). Estándares nacionales de tecnologías de información y comunicación (TIC) para docentes. EE. UU.:ISTE.

Ministerio de Educación de Chile. (2011). Competencias y Estándares TIC para la profesión docente. Santiago de Chile.

Ministerio de Educación Nacional. (2013). Competencias TIC para el desarrollo profesional docente. Bogotá: MEN

O'reilly, T. (2007). What Is Web 2.0. Design Patterns and Business Models for the Next Generation of Software. Communications \& Strategies, 65, 17- 
37.

Ortiz de Zarate, A. (2008). Manual de uso del blog en la empresa. Cómo prosperar en la sociedad de la conversación. Barcelona: Zero Factory, S. L

Prendes, M.P. (Dir.) (2010). Competencias TIC para la docencia en la universidad pública española: Indicadores y propuestas para la definición de buenas prácticas. Informe del proyecto financiado por la Secretaría de Estado de Universidades e Investigación del Ministerio de Educación dentro de la convocatoria Estudio y Análisis (EA20090133).

Ramírez, Y. V. y Peña, J. B. (2011). La Web 3.0 como Herramienta de Apoyo para la Educación a Distancia. Eticanet, 9(10).

Ribes, X. (2007). La Web 2.0. El valor de los metadatos y de la inteligencia colectiva. TELOS: Cuadernos de Comunicación e Innovación, 73.

Salganik L.H., Rychen D.S., Moser U. \& Konstant J.W. (1999). Definición y selección de competencias. Proyectos sobre Competencias en el Contexto de la OCDE.Análisis de base teórica y conceptual.

Santos, A. L., Nava, M. X. y Godoy, D. A. (2009). Web 3.0: integración de la Web Semántica y la Web 2.0 (informe). Recuperado de http://www. albertolsa.com/2009/07/30/web-30-integracionde-la-web-semantica-y-la-web-20/

UNESCO (2011). UNESCO ICT competency framework for teacher. Eduteka.

UNESCO. (2008). Estándares de competencias en TIC para docentes. Eduteka.

Universidad de Murcia. (2011). Proyecto de acreditación de competencias TIC del alumnado de la UM. Murcia: Universidad de Murcia. 